\title{
Consumo de drogas entre los Especialistas Internos Residentes de Jaén (España) y su relación con el consejo clínico
}

\section{Drugs consumption among Intern Specialists in Jaén (Spain) and their relationship to clinical counseling}

\author{
Francisco Javier Valverde Bolívar*; Alejandro \\ Pérez Mllena**; Andrés Moreno Corredor*
}

*Unidad Docente de Medicina Familiar y Comunitaria de Jaén. Servicio Andaluz
de Salud.
${ }^{* \star}$ Centro de Salud El Valle (Jaén). Servicio Andaluz de Salud.
Enviar correspondencia a:
Francisco Javier Valverde Bolívar
Unidad Docente de Medicina Familiar y Comunitaria de Jaén.
Complejo Hospitalario de Jaén.
C/ Avenida Ejercito Español número 14. CP 23007. Jaén.
Teléfono 953008078
Correo electrónico: franciscoj.valverde.sspa@juntadeandalucia.es

recibido: Septiembre 2012 aceptado: Febrero 2013

\section{Resumen}

Hay pocos estudios sobre prevalencia de consumo de alcohol, tabaco y drogas ilegales entre los especialistas internos residentes (EIR) o sobre el empleo que hacen del consejo clínico. Se diseña un estudio multicéntrico transversal mediante cuestionario validado y autoadministrado via correo electrónico, para describir el consumo de los EIR de 17 centros docentes de una provincia andaluza (4 hospitales, 13 centros de salud) y su relación con el consejo. Participan 215 EIR (81\% de cuestionarios válidos); edad media 31,2 años $( \pm 0,7), 70 \%$ mujeres, 13\% extranjeros, sólo 6\% enfermería. Un $78 \%$ consumen alcohol (edad inicio 16,8 años $\pm 0,3$ ), 81\% de forma ocasional y $17 \%$ fin de semana. La ingesta alcohólica semanal es 5,9 UBE $( \pm 5,8)$, fundamentalmente cerveza y combinados; 17\% con patrón binge-drinking, mayor en hombres ( $p=, 001$ X2). Un 19\% fuman, de ellos un 71\% diariamente con una media de $8,9 \pm 1,6$ cigarrillos/dia; la dependencia es baja $(68 \%)$ y dos terceras partes han intentado dejar de fumar. Sólo un 3\% consumen cannabis. La quinta parte de los EIR no suelen aconsejar contra el consumo de tabaco (21\%), la tercera parte no aconseja contra el alcohol $(34 \%)$ y casi la mitad tampoco aconseja sobre drogas $(44 \%)(p=, 001 \times 2)$. Mediante regresión logística se aprecia un mayor consejo clínico entre los EIR de mayor edad. El consejo contra el alcohol es mayor entre mujeres (OR $2,93)$ y, posiblemente, en los que no tienen un consumo tipo binge-driking (OR 2,32). El comienzo tardío del tabaquismo implica un menor consejo contra las drogas ilegales $(O R$ 0,76).

Palabras claves: internado y residencia, internado no médico, alcohol, tabaquismo, droga ilegal, consejo dirigido.

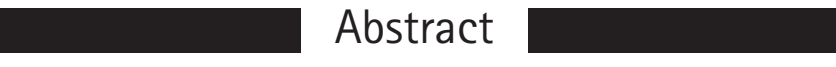

There are few studies on the prevalence of alcohol, tobacco and illegal drugs consumption among Specialist Interns (EIR) and their counseling to the patients. A multicenter cross-sectional study is carried out, consisting in a self-administered validated questionnaire to describe the consumption of the EIR of 17 health centers in Jaén (Andalusia) (4 hospitals, 13 primary care) and their relationship with their counseling. 215 EIR participate with $81 \%$ of valid questionnaires: mean age 31,2 years $( \pm 0,7), 70 \%$ women, $13 \%$ foreigners, only $6 \%$ nursing. Of them 78\% consumed alcohol (onset age $16,8$ years $\pm 0,3), 81 \%$ occasionally and $17 \%$ weekend. The alcohol average weekly intake was $5,9( \pm 5,8) \cup B E$, especially beer and cocktails; $17 \%$ show a binge-drinking pattern (more frequent in men, $p=, 001 \times 2$ ). 19\% smoke. A total of $71 \%$ smoke on a daily bases (mean of $8,9 \pm 1,6$ cigarettes/day); the nicotine dependence is low (68\%) and two thirds have tried to quit. Only 3\% use cannabis. A fifth part of EIR does not usually advise against smoking use (21\%), a third part does not advise against alcohol (34\%) and almost half of them neither advises against drugs (44\%) $(p=, 001 \times 2)$. Logistic regression showns greater clinical advice from older EIR. Advice against drinking alcohol provided by EIR women is more frequent $(O R 2,93)$ and, probably, even more in EIR that binge drink (OR 2,32). Late smoking onset is related to less clinical advice against illegal drugs $(O R, 76)$.

Key words: adolescents, alcohol use disorders, alcohol abuse, alcohol dependence, gender differences. 
$\mathrm{E}$ consumo de tabaco y alcohol en las sociedad europea y española sigue presente pese al conocimiento general de sus efectos nocivos y la mayor percepción del riesgo para todas las conductas de consumo de drogas, tanto para el consumo ocasional como para el habitual (Observatorio Español sobre Drogas, 2009). Según la Encuesta Europea de Salud de 2009 (Instituto Nacional de Estadística, 2009) más de la cuarta parte de la población mayor de 16 años fumaba a diario, con una prevalencia superor entre hombres frente a mujeres (31\% por $21 \%)$, teniendo más de la mitad una dependencia baja a la nicotina pero con una inmensa mayoría en estadios precontemplativos respecto al abandono (Fu et al., 2011). El consumo de alcohol es en su mayoría ocasional (39\%) con una menor proporción de consumo diario (13\%). Las prevalencias de consumo de tabaco y de alcohol han disminuido significativamente en la población española desde el inicio del siglo XXI (Observatorio Español sobre Drogas, 2009) y se mantienen estables desde el año 2006, pero parece que aumenta la tendencia a beber de forma más intensiva entre los consumidores de alcohol (Instituto Nacional de Estadística, 2009; Observatorio Español sobre Drogas, 2009), al igual que ocurre con los adolescentes (Observatorio Español sobre Drogas, 2010). En la población de estudiantes de 14 a 18 años el consumo es mayoritariamente experimental u ocasional, vinculado al ocio y al fin de semana y con un aumento progresivo con la edad (Observatorio Español sobre Drogas, 2010).

No parece existir un consumo de drogas diferenciado para los profesionales sanitarios (Kaneita, Uchida, y Ohida, 2010; Smith y Leggat, 2007; Voigt et al., 2009), con unas prevalencias de consumo similares, incluidas drogas ilegales, pese a tener un mayor conocimiento sobre los peligros de su uso y su especial labor de promoción y prevención. Así, los estudiantes de medicina presentaron un porcentaje de fumadores superior a la población general (entre el 22\% y el 29\%) (La Torre et al., 2012; Voigt et al., 2009), un consumo de alcohol frecuente que llega a superar, en algunos estudiantes masculinos, los 60 gramos diarios, y un uso de drogas ilegales elevado (40\% en hombres y $15 \%$ en mujeres). Hay menos estudios sobre médicos residentes, mostrando un consumo frecuente y regular de alcohol y tabaco, y en menor proporción marihuana (Handel, Raja, y Lindsell, 2006; Hughes, Conard, Baldwin, Storr, y Sheehan, 1991; La Torre et al., 2012; McBeth et al., 2008). En España (Blancafort, Masachs, Valero, y Arteman, 2009) se ha encontrado un consumo de tabaco entre los especialistas internos residentes (EIR) de un $30 \%$, sin diferencias por sexos y con una edad media de inicio de 19 años, un consumo de sustancias psicoactivas en el último mes cercano al $4 \%$ y un bajo consumo de alcohol.

La formación de los EIR incluye la realización de diferentes programas de actividades preventivas, como el indagar de forma bianual por el consumo de tabaco y alcohol de los pacientes (Cabezas Peña et al., 2007). Hay suficientes evidencias que demuestran cómo el consejo contra el tabaco y contra el alcohol consiguen reducir las prevalencias de su consumo (Sullivan, Tetrault, Braithwaite, Turner, y Fiellin, 2011). Pese a ello, este consejo clínico es inferior al $40 \%$ de las consultas realizadas en la mayoría de los estudios (Ballbè et al., 2009; Rodríguez Ibáñez et al., 2001; Wallace, Sairafi, y Weeks, 2006) con una intervención posterior mucho menor. Diversos facto- res influyen en la realización del consejo contra el consumo de drogas: el sexo (las mujeres proporcionan más consejo), la especialidad (aconsejan más los médicos generales o los internistas que los pediatras), la edad, la situación geográfica (se ofrece más consejo en el ámbito urbano) y el propio consumo del profesional (Tremblay et al., 2001).

Existen pocos estudios sobre el consumo de drogas de los EIR que incluyan personal de enfermería, farmacia o psicología, y menos aún que relacionen este consumo con la presencia del consejo contra el consumo de drogas en las actividades clínicas. Por ello, el presente trabajo se plantea como objetivo describir los hábitos de consumo de alcohol, tabaco y drogas ilegales de los EIR de una provincia andaluza y determinar si el consumo personal condiciona los consejos dados a los pacientes en la actividad clínica cotidiana.

\section{Métodos}

\section{Diseño y población}

Se diseña un estudio multicéntrico descriptivo transversal mediante encuesta. La población de estudio está compuesta por todos los especialistas internos residentes (EIR) que estaban cursando una especialidad en la provincia de Jaén durante el año 2011, un total de 215 residentes, pertenecientes a los 4 hospitales y 13 centros de salud docentes de la provincia. Los criterios de inclusión fueron encontrarse en situación activa en el momento de la encuesta y estar dado de alta en PORTALEIR, que es la página web para el seguimiento y la formación de los EIR en Andalucía (www.portaleir.es). Se excluyeron aquellos profesionales que no desearon participar y los cuestionarios que no estaban totalmente cumplimentados.

\section{Variables}

Se utiliza una encuesta autoadministrada previamente validada (Pérez Milena et al., 2007) que contiene un total de 26 preguntas dividida en cuatro apartados. Los datos demográficos incluyen como variables la edad (en años), el sexo, el país de origen, la especialidad que se cursa en el momento de completar el cuestionario y el año de residencia. El segundo bloque indaga sobre datos de consumo, dependencia y motivación para el abandono del hábito tabáquico: cantidad de tabaco fumado (expresado en cigarrillos al dia), patrón de consumo, la edad de inicio y de cese (si lo hubo), así como el test de Fagerström para analizar el grado de dependencia hacia la nicotina y el número de intentos para dejar de fumar, valorando la fase en la que se encuentra el EIR dentro del ciclo de cambio de Proschaka. Para analizar los estadios del cambio respecto al tabaco, se consideró como precontemplación cuando no desea dejar de fumar, contemplación cuando se plantea el cambio en los próximos 6 meses y acción cuando desea el cese del tabaquismo en los próximos 30 días (Becoña y Lorenzo, 2004). Para medir la dependencia mediante el test de Fagerström (puntuación de la escala entre 0 y 11), se consideró un valor igual o mayor a siete como nivel alto de dependencia, mientras que uno menor de tres como una dependencia baja (Becoña, Gómez-Durán, Álvarez- 
Soto y García, 1992). El tercer bloque incluye datos sobre el consumo de alcohol, tanto el patrón como la cantidad medido como unidades de bebida estándar (UBE) a través del número de distintas bebidas alcohólicas ingeridas por semana. Se consideró binge drinking aquel consumo concentrado episódico de alcohol $\geq 5$ UBE para hombres $y \geq 4$ para mujeres. También se incluyó el consumo de otras drogas ilegales, tanto el tipo como el patrón de consumo. Este patrón de consumo se homogeneizó para todas las sustancias, siendo recogido como consumo intermitente (esporádico o de forma ocasional), en fin de semana (o asociado a días festivos) y diario (Pérez Milena et al., 2007). El cuestionario recoge, finalmente, la frecuencia de utilización del consejo clínico contra el consumo de estas sustancias a los pacientes atendidos en las tareas clínicas cotidianas, entendiendo por tal cualquier tipo de pregunta sobre el consumo y/o de intervención sobre el mismo. La respuesta se recoge en cuatro categorías para cada tipo de sustancia consumida: nunca/casi nunca, a veces, a menudo y casi siempre/siempre.

El cuestionario fue difundido a través de un correo electrónico personal a cada EIR, invitándole a participar, explicándole el propósito del estudio, asegurándole el carácter privado de sus respuestas y su explotación de forma global. En ese mismo correo disponían de un enlace directo que les dirigian al cuestionario, emplazado en una página web personalizada (dentro del dominio www.e-encuesta.com). Para minimizar las pérdidas se reenvió la encuesta en 3 ocasiones con una periodicidad quincenal. Se consideró que una encuesta es válida cuando sus ítems habían sido cumplimentados en un 100\%, con criterio de un mínimo de un $60 \%$ de cuestionarios rellenados del total de las encuestas enviadas para conseguir una validez adecuada de los resultados.

En referencia a la especialidad cursada se agrupó en cuatro grandes bloques según la afinidad del programa formativo de las mismas: Medicina Familiar (44\% del total), Médicas Hospitalarias (37\%) [17\% Pediatría, 14\% Anestesiología y Reanimación, 15\% Medicina Interna, 9\% Psiquiatría, 6\% Hematología/ Radiodiagnóstico/Análisis Clínicos, 5\% Cardiología, 4\% Medicina Intensiva/Psicología Clínica, 3\% Aparato Digestivo/ Endocrinología y Nutrición/Medicina del trabajo, 2\% Nefrología/ Oncología Médica], Quirúrgicas Hospitalarias (12\%) [33\% Cirugía Ortopédica y Traumatología/Obstetricia y Ginecología, 14\% Oftalmología, 10\% Cirugía General y del Aparato Digestivo/Otorrinolaringología] y Enfermería (7\%) [75\% Enfermería Obstétrica, 17\% Farmacología y 8\% Enfermería del Trabajo].

\section{Análisis estadístico}

Se emplea el programa SPSS (15.0) para comprobar la normalidad de los datos y realizar un análisis descriptivo de los mismos, expresando frecuencias (absolutas y porcentaje) para variables cualitativas y medias y desviación típica para cuantitativas. En el análisis bivariante se fijó la significación estadistica en $p<, 05$, empleándose la prueba de $\chi^{2}$ para variables cualitativas, y la $t$ de Student (o ANOVA) para la comparación de medias. Finalmente, se realizó mediante el programa $R$ commander un análisis multivariante mediante regresión logística binaria bivariada inicialmente y posteriormente multivariante por pasos hacia atrás con criterio Razón de Verosimilitud (RV), entendiendo como variable dependiente el consejo contra el consumo de las tres sustancias, siendo el resto de las variables independientes. En la regresión se incluyó las variables con $p$-valor $<, 25$ en el test de Wald. La codificación realizada ha sido: consejo casi siempre/siempre $=1$; consejo nunca/a veces $=0$. Las categorías de referencia para codificar las variables dummy han sido elegidas automáticamente por el programa. Por último, el ajuste del modelo fue valorado con el test de Hosmer-Lemeshow.

\section{Aspectos éticos}

El proyecto de investigación fue aprobado por la Comisión de Ética e Investigación Sanitarias del Hospital de referencia (según normativa del RD 223/04). El tratamiento de los datos de carácter personal de los sujetos que participaron en el estudio se ajustó a lo establecido en la Ley Orgánica de Protección de Datos de Carácter Personal, 15/1999 de 13 de Diciembre. Con los datos obtenidos se crearon dos bases de datos para el análisis estadístico, una con la identificación personal de los EIR, solo accesible a dos investigadores, y otra con el resto de variables codificadas por claves para proteger el anonimato.

\section{Resultados}

La población total de estudio comprende a 215 especialistas internos residentes (EIR), de los que se obtiene un total de 174 cuestionarios válidos ( $81 \%$ del total). La media de edad es de 31,2 años $( \pm 7,2)$, siendo superior en los EIR de medicina familiar (tabla 1) $(F=14,197 ; p=, 001$ test ANOVA). Un 70\% son mujeres. La mayoría son españoles (87\%) frente a los procedentes de centro y Sudamérica (11\%), norte de África (2\%) y resto de Europa (1\%). Hay una distribución casi homogénea en los cuatro primeros años de estudios: $31 \%$ en primer año, 25\% en segundo, $22 \%$ en tercero y $21 \%$ en cuarto; sólo dos EIR de $5^{\circ}$ año responden a la encuesta. Las principales variables sobre el consumo de sustancias tóxicas en función de la especialidad cursada se exponen en la tabla 1. Sólo 32 EIR no consumen ningún tipo de sustancia (19\% del total).

\section{Consumo de alcohol}

Un 78\% consumen alcohol, en mayor proporción en los EIR de medicina y de cirugía hospitalarias (tabla 1$)\left(\mathrm{Ji}^{2}=13,389\right.$; $p=, 010$ test $x^{2}$ ). La edad de inicio para el consumo es de 16,8 años $( \pm 3,0)$. El patrón de consumo más frecuente es el intermitente $(81 \%)$ seguido del consumo en fin de semana (18\%) (figura 1). Hay un $17 \%$ de consumo tipo binge-drinking, menos frecuente en los EIR de medicina familiar e inexistente en enfermería (figura 2$)\left(\mathrm{Ji}^{2}=30,144 ; \mathrm{p}=, 001\right.$ test $\left.\mathrm{X}^{2}\right)$; comparando por sexos, es más frecuente en hombres (26\%) que en mujeres $(12 \%)\left(\mathrm{Ji}^{2}=34,238 ; p=, 001\right.$ test $\left.\mathrm{X}^{2}\right)$. La cerveza es la bebida más consumida $(2,7 \mathrm{UBE} /$ semana $\pm 2,8)$, con un consumo semanal medio de $5,9 \operatorname{UBE}( \pm 5,8)$, que es superior en los EIR de cirugía hospitalaria debido a un mayor consumo de bebidas de alta graduación (tabla 1) $(F=10,704 ; p=, 001$ test ANOVA). También hay diferencias en el consumo cuantitativo según el sexo, siendo superior en hombres $(7,8 U B E \pm 8,3)$ frente a mujeres $(4,9 U B E \pm 3,5)(t=2,511 ; p=, 013$ test $t$ Student). 
Tabla 1

Principales resultados sobre el consumo de alcohol, tabaco y drogas legales según la especialidad cursada

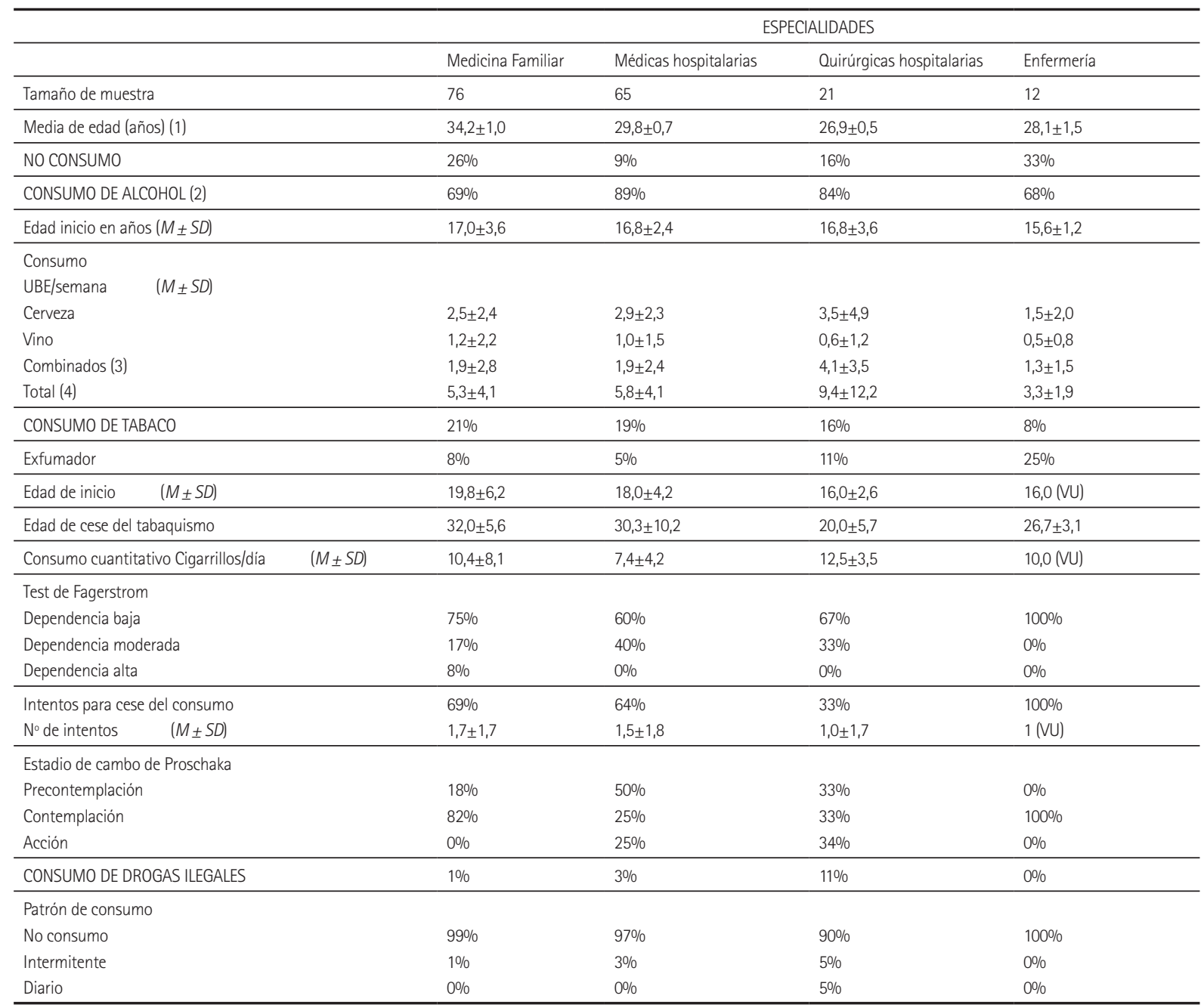

Los datos se muestran como medias con su desviación estándar. (VU) valor único

(1) $F=14,197 ; p=, 001$ test ANOVA (2) $\mathrm{Ji}^{2}=13,389 ; p=, 010$ test $\mathrm{X}^{2}(3) \mathrm{F}=8,199 ; \mathrm{p}=, 001$ test ANOVA (4) $\mathrm{F}=10,704 ; \mathrm{p}=, 001$ test ANOVA.

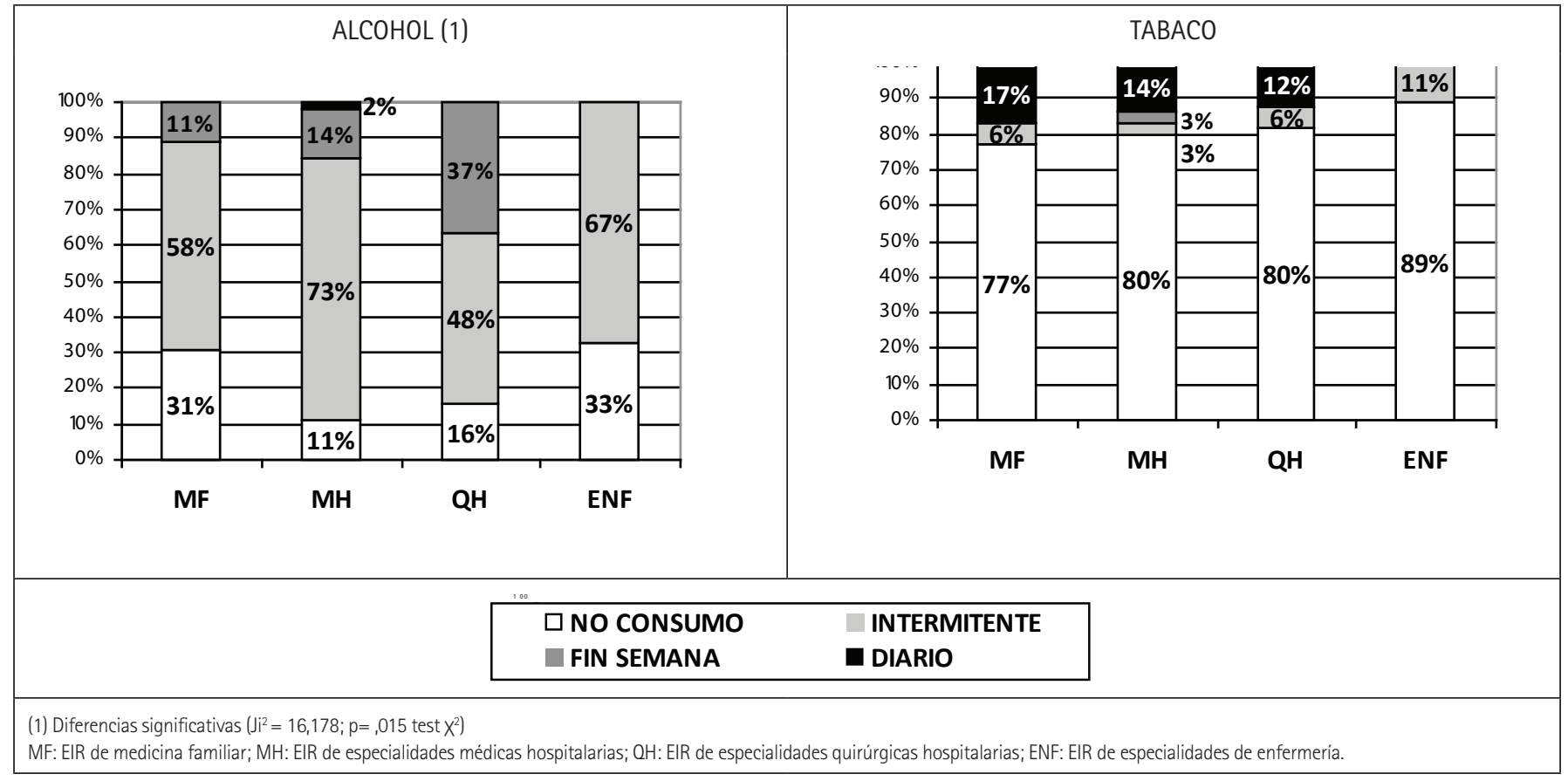

Figura 1. Patrón de consumo de alcohol y tabaco según la especialidad cursada 


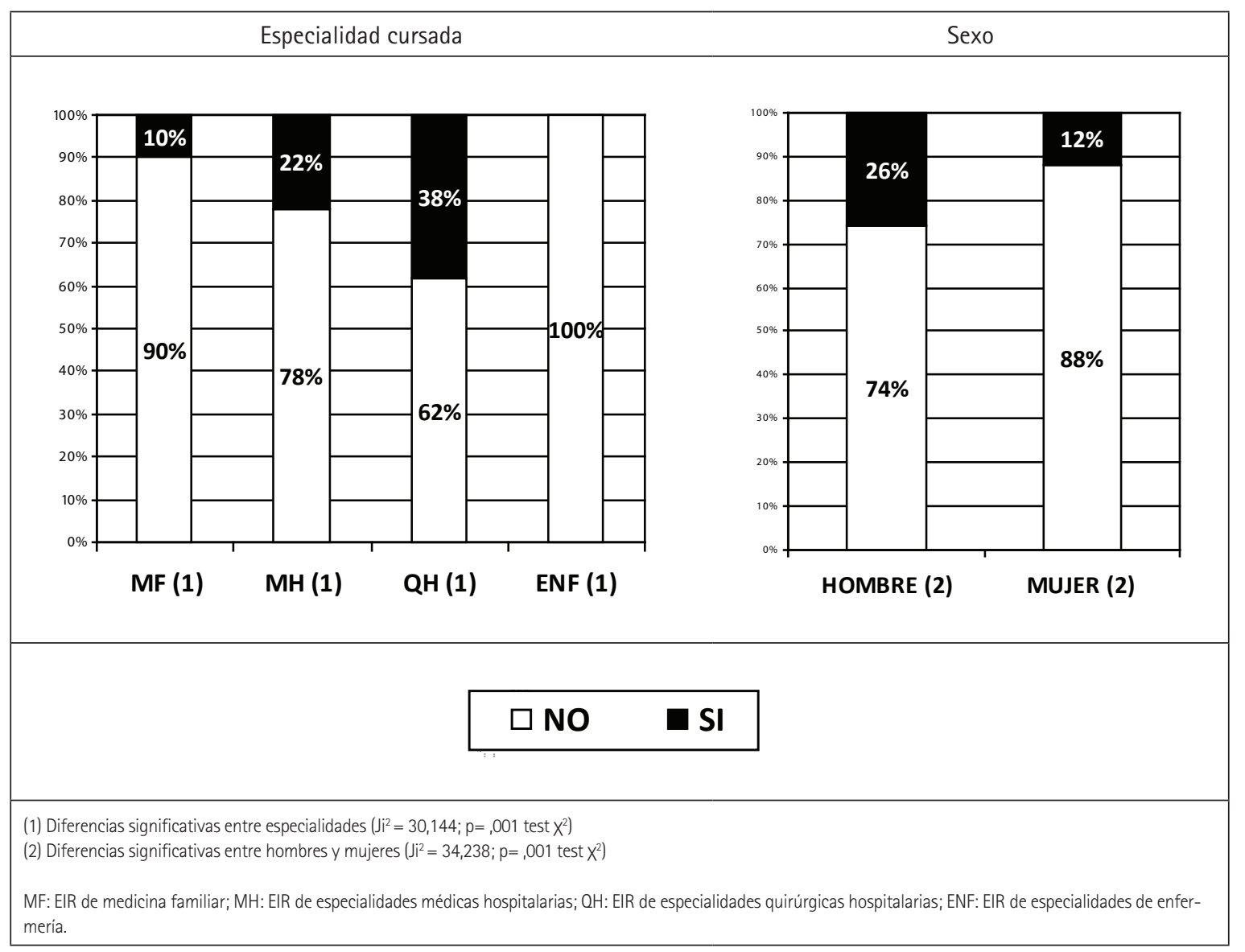

Figura 2. Distribución del fenómeno del binge drinking según la especialidad cursada y el sexo del EIR.

\section{Consumo de tabaco}

El 19\% de los encuestados fuman, en su mayoría de forma diaria (71\%), con una quinta parte de consumo intermitente (22\%) (figura 1). La media de la edad de inicio del tabaquismo se sitúa en 18,6 años $( \pm 5,1)$ y el consumo medio es de 9,3 años $( \pm 6,4)$. Según el test de Fagerström, un 68\% presentaron una dependencia baja a la nicotina mientras que sólo el $4 \%$ puntuaron como dependencia elevada. Un 9\% del total de los encuestados son exfumadores y dejaron de fumar con una media de edad de 28,8 años $( \pm 7,1)$; entre los fumadores, un $64 \%$ han hecho una media de 1,6 intentos $( \pm 1,6)$ para dejar de fumar. Según los estadios de cambio de Proschaka, un 33\% se encuentran en precontemplación, un 55\% en contemplación y el restante $15 \%$ en preparación para dejar de fumar.

\section{Consumo de drogas ilegales}

Sólo un 3\% reconoce consumir drogas ilegales, la mayoría de forma intermitente (figura 1). No existe relación entre los patrones de consumo de alcohol, tabaco y drogas ilegales. Sin embargo, los fumadores que no consumen alcohol tienen un consumo de cigarrillos diarios superior a los que sí beben (13,3 $\pm 11,9$ frente a $8,6 \pm 4,9)(t=3,171 ; p=, 001$ test $t$ Student $)$. Por otra parte, los EIR fumadores consumen más cantidad de alcohol de alta graduación que los no fumadores (3,8 UBE semanales $\pm 3,4$ frente a $1,8 \pm 2,4)(t=9,220 ; p=, 001$ test $t$ Student $)$.

\section{Características del consejo contra el uso de drogas}

El consejo en consulta sobre el consumo de alcohol se ofrece siempre o casi siempre en un 66\% de los EIR encuestados, aumentando al $79 \%$ en el caso del tabaco y disminuyendo al 56\% para las drogas ilegales. Existen diferencias significativas según la especialidad cursada siendo más frecuente el consejo para todas las drogas en las especialidades de medicina familiar y enfermería frente a las especialidades hospitalarias (figura 3) ( $p<0,05$ test $x 2$ ). Estas diferencias también se aprecian en el sexo, con un mayor consejo por parte de las mujeres acerca del alcohol ( $73 \%$ y $51 \%)\left(\mathrm{Ji}^{2}=2,783 ; p=, 05\right.$ test $\left.\mathrm{X}^{2}\right)$ y de las drogas ilegales (61\% y 44\%) $\left(\mathrm{Ji}^{2}=2,391 ; p=, 05\right.$ test $\left.\mathrm{X}^{2}\right)$.

Con el propósito de identificar si existe una asociación entre el consejo en consulta sobre alcohol, tabaco y drogas ilegales por parte de los EIR y las diferentes variables del consumo de estas sustancias, se realizaron análisis de regresión logística para cada una de las variables dependientes agrupadas como consejo no (categorías nunca y a veces) y sí (casi siempre y siempre). Los modelos obtenidos se muestran en la tabla 2. La edad se constituye como una variable asociada en todos los modelos presentados, influyendo sobre la frecuencia del consejo dado por los EIR para las tres sustancias estudiadas, de forma que a mayor edad del sanitario hay una mayor presencia del consejo. El sexo, en cambio, sólo se relaciona con el consejo contra el alcohol de forma que ser mujer condiciona una probabilidad casi tres veces mayor de ofertar consejo en 


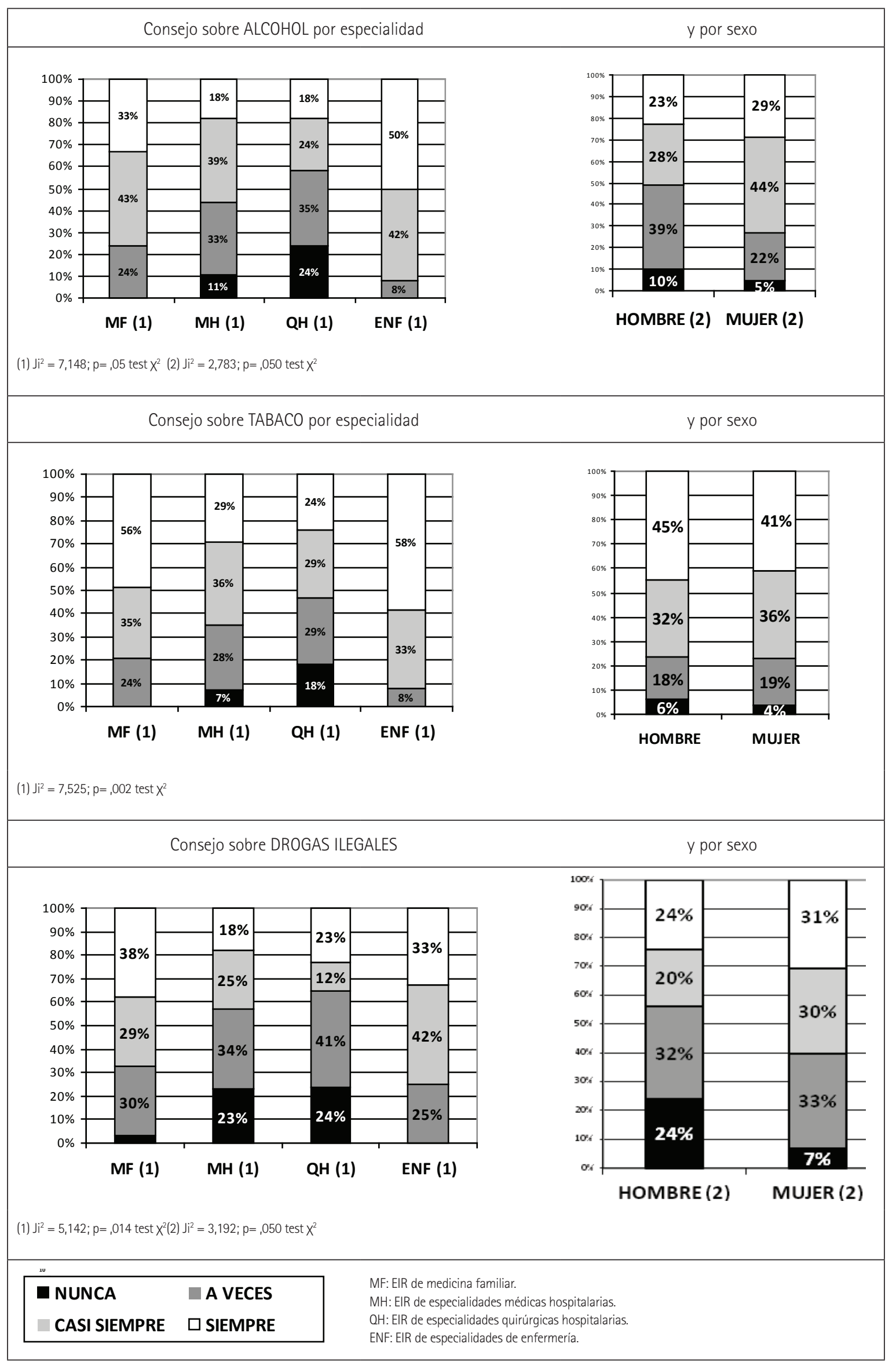

Figura 3. Frecuencia del consejo sobre alcohol, tabaco y drogas ilegales ofertados a los pacientes por los ElR según la especialidad cursada y el sexo. 
Tabla 2

Modelos del análisis de Regresión Logística para la frecuencia del consejo sobre alcohol, tabaco y drogas como variables dependientes frente a las características del consumo de dichas sustancias

\begin{tabular}{|c|c|c|c|c|c|}
\hline \multicolumn{6}{|l|}{ Consejo sobre alcohol } \\
\hline Variable & Coeficiente & $O R$ & Intervalo de confianza 95\% & Chi2 & Nivel de significación \\
\hline Edad & 0.066 & 1.06 & {$[1.01 ; 1.14]$} & 2.088 & 0.03682 \\
\hline Sexo (mujer) & 1.077 & 2.93 & {$[1.33 ; 6.61]$} & 2.653 & 0.00799 \\
\hline Binge-drinking & -0.828 & 0.43 & {$[0.16 ; 1.14]$} & -1.691 & 0.09078 \\
\hline Consumo de alcohol & 0.176 & 1.19 & {$[0.33 ; 3.89]$} & 0.285 & 0.77583 \\
\hline \multicolumn{6}{|l|}{ Consejo sobre tabaco } \\
\hline Variable & Coeficiente & $O R$ & Intervalo de confianza 95\% & Chi2 & Nivel de significación \\
\hline Edad & 0.150 & 1.16 & {$[1.05 ; 1.32]$} & 2.621 & 0.00877 \\
\hline Sexo (mujer) & 0.416 & 1.52 & {$[0.54 ; 4.15]$} & 0.810 & 0.41773 \\
\hline Número de cigarrillos & 0.065 & 1.07 & {$[0.89 ; 1.32]$} & 0.664 & 0.50664 \\
\hline Consumo de tabaco & -1.534 & 0.22 & {$[0.03 ; 1.52]$} & -1.509 & 0.13131 \\
\hline \multicolumn{6}{|l|}{ Consejo sobre drogas ilegales } \\
\hline Variable & Coeficiente & $O R$ & Intervalo de confianza 95\% & Chi2 & Nivel de significación \\
\hline Edad & 0.206 & 1.23 & {$[1.05 ; 1.55]$} & 2.161 & 0.0307 \\
\hline Sexo (mujer) & 0.986 & 2.68 & {$[0.55 ; 15.47]$} & 1.185 & 0.2362 \\
\hline $\begin{array}{l}\text { Edad de inicio del tabaquis- } \\
\text { mo }\end{array}$ & -0.268 & 0.76 & {$[0.55 ; 0.98]$} & -1.867 & 0.0618 \\
\hline Número de cigarrillos & -0.020 & 0.98 & {$[0.81 ; 1.19]$} & -0.215 & 0.8301 \\
\hline
\end{tabular}

cada acto clínico frente a los hombres. Sobre el consejo contra el alcohol se observa una relación con la presencia de un consumo tipo binge-drinking del propio EIR, de forma que incrementa de forma casi significativa el riesgo de no dar consejo sobre el alcohol entre los que consumen alcohol de esta forma. Por último, la edad de comienzo del tabaquismo se asocia al consejo sobre drogas ilegales: cada año más en la edad de inicio del consumo de tabaco implica una odds 1,3 veces mayor de no dar consejo sobre las drogas ilegales por parte del EIR.

\section{Discusión}

En este estudio, el consumo de drogas en la población EIR presenta diferencias con respecto a la población general (Observatorio Español sobre Drogas, 2009). La prevalencia de consumo de alcohol es mayor, con un patrón de consumo ligado al ocio y una alta presencia de un consumo tipo binge-drinking, aunque con una media de edad de inicio mucho más elevada. El consumo de tabaco es inferior a la población general con un señalado porcentaje de exfumadores y un gran número de fumadores motivados para el cese del consumo. Las drogas ilegales son poco consumidas, destacando al igual que en la población adolescente el consumo de cannabis.

La oferta de consejo clínico en las actividades asistenciales de los EIR es bastante frecuente, por encima de los porcentajes obtenidos en otros estudios (Ballbè et al., 2009), influenciado con la edad (para todas las drogas) y el sexo femenino (para el alcohol) pero sin relación con el consumo personal. Hay una mayor presencia del consejo antitabaco, una intermedia sobre el consumo de alcohol y una baja frecuencia de recomendaciones sobre las drogas ilegales. Este hallazgo puede deberse a la misma presión social que normaliza más el consumo de alcohol frente al tabaco, junto al temor a invadir la privacidad del paciente al preguntar por drogas ilegales.

Los datos obtenidos sobre la prevalencia del consumo del alcohol coinciden con otros resultados obtenidos en estudiantes de Medicina (Voigt et al., 2009), en médicos residentes (Hughes et al., 1991; Martínez, Medina-Mora, y Rivera, 2004; McBeth et al., 2008; Pérez Milena et al., 2010) y médicos adjuntos (Kenna y Wood, 2005; Rodríguez Fernández, Espí Martínez, Canteras Jordana, y Gómez Moraga, 2001; Rosta y Aasland, 2010). El patrón de consumo es de tipo anglosajón, ligado al ocio y relacionado con el modelo binge-drinking, de forma superior a la población general y a los médicos adjuntos (Blancafort et al., 2009; Hull, DiLalla, y Dorsey, 2008; Rosta, 2008; Voigt et al., 2009) pero similar al de la población joven (Observatorio Español sobre Drogas, 2010; Pérez Milena et al., 2007) y al de los estudiantes varones de medicina, donde un $30 \%$ pueden superar un consumo de 60 gramos por ocasión de consumo (Hull et al., 2008; Voigt et al., 2009).

Esta forma de beber se relaciona con una menor oferta de consejo contra el alcohol y con la especialidad cursada, preferentemente las especialidades médicas hospitalarias $y$, sobre todo, quirúrgicas (Rosta et al., 2005, 2010). La posible relación del sexo masculino con un menor consejo contra el alcohol (Cerrada, Olmeda, Senande, Rodríguez, y Sanz Cuesta, 2005; Sreeramareddy et al., 2010) podría estar mediada por una mayor presencia de consumo tipo binge-drinking en los varones, lo que puede normalizar y banalizar la situación de consumo de los pacientes atendidos. 
La prevalencia del consumo de tabaco es inferior a la población general (Instituto Nacional de Estadística, 2009; Observatorio Español sobre Drogas, 2009) y también a otras series estudiadas en EIR, que alcanzan hasta la mitad de la población de residentes (Blancafort et al., 2009; Ferrero, Castaños, Durán, y Blengini, 2004; Martinez et al., 2004). Sin embargo, presenta características muy similares a otras poblaciones como enfermeros o estudiantes de medicina (0'Donovan, 2009; La Torre et al., 2012; Voigt et al., 2009). Las causas de estas diferencias puede hallarse en la variabilidad introducida por las diferentes políticas sanitarias, etapas culturales y de concienciación ciudadana en las que cada sociedad se encuentre: Así, se obtienen prevalencias muy dispares en estudios sobre médicos adjuntos: desde un consumo del 4\% en Estado Unidos hasta un 40\% en Bosnia o Turquía (Smith et al., 2007).

Parece que, al igual que en los adolescentes (Pérez-Milena et al., 2006), hay una magnífica oportunidad para el cese del tabaquismo en la etapa EIR ya que hasta las tres cuartas partes de los fumadores han intentado dejar de fumar o están pensando en dejarlo. Los niveles de dependencia a la nicotina de los EIR son similares a los encontrados en la población general (Fu et al., 2011).

El escaso pero preocupante consumo de drogas ilegales puede deberse a un sesgo de deseabilidad, aunque coincide con datos recogidos de otros sanitarios (Kenna et al., 2005). En general los estudios presentan prevalencias muy dispares de consumo de drogas ilegales en facultativos (Hughes et al., 1991; Martinez et al., 2004; McBeth et al., 2008, 2009), lo que dificulta conocer el porcentaje real de consumo de drogas ilegales entre los sanitarios, aunque parece ser inferior al de la población general (Observatorio Español sobre Drogas, 2009). El consumo se centra en el uso del cannabis, predominando en hombres y siendo inferior al que presentan los estudiantes de medicina, que podría extenderse hasta una tercera parte de los universitarios (Voigt et al., 2009).

En general los consejos de los sanitarios tienen poca presencia en la actividad clínica cotidiana (Rodríguez Ibáñez et al., 2001; Talley et al., 2011; Wallace et al., 2006), incluso tras recibir una formación específica (Ballbè et al., 2009; Sreeramareddy et al., 2010). Existen barreras que dificultan el consejo clínico, tales como la percepción negativa de los profesionales, la baja receptividad de los pacientes o la falta de tiempo para su realización (Cerrada et al., 2005). Los resultados obtenidos indican la ausencia de relación entre el consumo personal de los EIR y la emisión de consejos contra el consumo de drogas (Ferrero et al., 2004; Tremblay et al., 2001) a diferencia de otros estudios en enfermería (O'Donovan, 2009). Sólo el patrón de consumo de alcohol y el inicio tardio del tabaquismo parecen tener cierta influencia (Rosta et al., 2005, 2010). La presencia de una mayor edad condicionaría mayor frecuencia del consejo, mientras que el sexo no parece influir o (Hull et al., 2008; Cerrada et al., 2005).

Hay pocos estudios sobre los hábitos de consumo de drogas y la relación con el consejo clínico entre los especialistas internos residentes (Blancafort et al., 2009; Ferrero et al., 2004; Hughes et al., 1991; McBeth et al., 2008), por lo que este estudio puede aportar un referente para otras poblaciones de EIR a nivel nacional. Aunque los resultados pueden estar influenciados por los sesgos de respuesta y memoria, parecen ser bastantes representativos dado el bajo número de pérdidas en el estudio frente a otros similares (Blancafort et al., 2009; McBeth et al., 2008) y tratarse de un cuestionario previamente validado con una alta accesibilididad y confidencialidad (Pérez Milena et al., 2007).

El profesional EIR presenta un consumo peculiar de drogas y su estancia formativa se convierte en un momento propicio para el abandono del consumo y para el aprendizaje de técnicas de promoción de la salud. La formación sobre el consejo clínico es una herramienta actualmente infrautilizada en el control y la prevención del consumo, con una implantación no sistemática sobre todo en la formación médica hospitalaria (Cerrada et al., 2005; Sreeramareddy et al., 2010). Es preciso que las actividades preventivas contra el consumo de drogas formen parte del currículum del EIR (Aalto, Pekuri, y Seppä, 2005; Ballbè et al., 2009), máxime cuando muchos pacientes esperan que el profesional sanitario aborde su consumo de una manera proactiva (Ulbricht et al., 2011). Se ha demostrado que esta formación en postgrado es eficaz independientemente de la formación de grado o de postgrado del EIR, pudiendo realizarse indistintamente en la consulta de medicina o de enfermería (Cabezas Peña et al., 2007; Scal, Hennrikus, Ehrlich, Ireland, y Borowsky, 2004). Los datos obtenidos plantean la necesidad de realizar estudios sobre la eficacia de las intervenciones contra el consumo de drogas en EIR y de la formación en técnicas específicas de consejo clínico.

\section{Reconocimiento}

Se agradece la ayuda prestada por María del Carmen Rosa Garrido, metodóloga de la Fundación de Investigación Biosanitaria Alejandro Otero de Jaén, por su ayuda en la elaboración de los resultados.

\section{Conflictos de intereses}

Este trabajo no presentó conflicto de intereses ni tiene ninguna conexión con la industria del tabaco, alcohol o industria farmacéutica u otras relaciones que puedan generar conflicto de intereses.

\section{Referencias}

Aalto, M., Pekuri, P. y Seppä, K. (2005). Implementation of brief alcohol intervention in primary health care: do nurses' and general practitioners' attitudes, skills and knowledge change? Drug and alcohol review, 24, 555-558. doi:10.1080/09595230500292904

Ballbè, M., Walther, M., Mondon, S., Nieva, G., Gual, A., Saltó, E. y Colom, J. (2009). Impacto de la formación en intervención breve. Diferencias en el abordaje hospitalario del consumo de tabaco y alcohol. Adicciones, 21, 113-118.

Becoña Iglesias, E., Gómez-Durán, B.J, Álvarez-Soto, E. y Garcia, MP. (1992). Scores of spanish smokers on Fagerström's Tolerance Questionnaire. Psychological Reports, 71, 1227-1233. 
Becoña Iglesias, E. y Lorenzo Pontevedra, M. C. (2004) Evaluación de la conducta de fumar. Adicciones, 16 (Supl. 2), 201-226.

Blancafort, X., Masachs, E., Valero, S. y Arteman, A. (2009). Estudio sobre la salud de los residentes de Cataluña. Barcelona: Fundación Galatea.

Cabezas Peña, C., Robledo de Dios, T., Marquésa, F., Ortega SánchezPinilla, R., Nebot Adella, M., Megido Badia, M. J.,...Prados Castillejo, J.A. (2007). Recomendaciones sobre el estilo de vida. Atención Primaria, 39 (Supp/3), 27-46.

Cerrada, E. C., Olmeda, C. L., Senande, E. B., Rodríguez, B. G. y Sanz Cuesta, T. (2005). Opiniones, prácticas, barreras y predisposición al cambio, a la hora de dar consejo para dejar de fumar. Atención Primaria, 36, 434-441. doi: 10.1157/13081057

Ferrero, F., Castaños, C., Durán, P. y Blengini, M. T. (2004). Prevalencia del cosumo de tabaco en médicos residentes de Pediatría en Argentina. Revista Panamericana de Salud Pública, 15, 395-399. doi:10.1590/ S1020-49892004000600005

Fu, M., Martínez-Sánchez, J. M., López, M. J., Nebot, M., Raich, A. y Fernández, E. (2011). Dependencia a la nicotina y preparación para dejar de fumar en la población española. Adicciones, 23, 103-109.

Handel, D. A., Raja, A. y Lindsell, C. J. (2006). The use of sleep aids among Emergency Medicine residents: a web based survey. BMC Health Services Research, 6, 136. doi:10.1186/1472-6963-6-136

Hughes, P. H., Conard, S. E., Baldwin, D. C., Storr, C. L. y Sheehan, D. V. (1991). Resident physician substance use in the United States. JAMA: the journal of the American Medical Association, 265, 2069-2073.

Hull, S. K., DiLalla, L. F. y Dorsey, J. K. (2008). Prevalence of healthrelated behaviors among physicians and medical trainees. Academic psychiatry: the journal of the American Association of Directors of Psychiatric Residency Training and the Association for Academic Psychiatry, 32, 31-38. doi:10.1176/appi.ap.32.1.31

Instituto Nacional de Estadistica (2009). Encuesta Europea de Salud (EES09). Madrid: Ministerio de Sanidad, Política Social e igualdad, España. Recuperado de http://www.msps.es/estadEstudios/ estadisticas/EncuestaEuropea/Principales_Resultados_Informe.pdf.

Kaneita, Y., Uchida, T. y Ohida, T. (2010). Epidemiological study of smoking among Japanese physicians. Preventive medicine, 51, 164167. doi:10.1016/j.ypmed.2010.04.015

Kenna, G. A. y Wood, M. D. (2005). The prevalence of alcohol, cigarette and illicit drug use and problems among dentists. Journal of the American Dental Association, 136, 1023-1032.

La Torre, G., Kirch, W., Bes-Rastrollo, M., Ramos, R. M., Czaplicki, M., Gualano, M.,...Boccia, A. (2012). Tobacco use among medical students in Europe: results of a multicentre study using the Global Health Professions Student Survey. Public health, 126, 159-164. doi:10.1016/j.puhe.2011.10.009

Martínez Lanz, P., Medina-Mora, M. E. y Rivera, E. (2004). Consumo de alcohol y drogas en personal de salud: algunos factores relacionados. Salud mental, 27, 17-27.

McBeth, B. D., Ankel, F. K., Ling, L. J., Asplin, B. R., Mason, E. J., Flottemesch, T. J. y McNamara, R. M. (2008). Substance use in emergency medicine training programs. Academic emergency medicine: official journal of the Society for Academic Emergency Medicine, 15, 45-53. doi:10.1111/j.1553-2712.2007.00008.x
McBeth, B. D., McNamara, R. M., Ankel, F. K., Mason, E. J., Ling, L. J., Flottemesch, T. J. y Asplin, B. R. (2009). Modafinil and zolpidem use by emergency medicine residents. Academic emergency medicine: official journal of the Society for Academic Emergency Medicine, 16, 1311-1317. doi:10.1111/j.1553-2712.2009.00586.x

Observatorio Español sobre Drogas de la Delegación del Gobierno para el Plan Nacional sobre Drogas. (2009). Encuesta Domiciliaria sobre Alcohol y Drogas en España (EDADES). Madrid: Ministerio de Sanidad Servicios Sociales e Igualdad, España. Recuperado de http://www.mspsi.es/gabinetePrensa/notaPrensa/pdf/ presentacionEdades200910.ppt.

Observatorio Español sobre Drogas de la Delegación de Gobierno para el Plan Nacional sobre Drogas. (2010) Encuesta Estatal sobre uso de Drogas en Enseñanzas Secundarias (ESTUDES). Madrid, Ministerio de Sanidad y Política Social, España. Recuperado de http://www. pnsd.msc.es/Categoria2/observa/pdf/Estudes2008.pdf.

O'Donovan, G. (2009). Smoking prevalence among qualified nurses in the Republic of Ireland and their role in smoking cessation. International nursing review, 56, 230-236. doi:10.1111/j.14667657.2008.00700.x

Pérez Milena, A., Javier Leal Helmling, F., Jiménez Pulido, I., Mesa Gallardo, I., Luz Martínez Fernández, M. y Pérez Milena, R. (2007). Evolución del consumo de sustancias tóxicas en los adolescentes de una zona urbana (1997-2004). Atención Primaria, 39, 299-304. doi:10.1157/13106285

Pérez Milena, A., Redondo Olmedilla, M., Mesa Gallardo, I., Jiménez Pulido, I., Martinez Fernández, M. L. y Pérez Milena, R. (2010). Motivaciones para el consumo de alcohol entre adolescentes de un instituto urbano. Atención Primaria, 42, 604-611. doi:10.1016/j. aprim.2009.12.009

Rodriguez Fernández, E., Espí Martínez, F., Canteras Jordana, M. y Gómez Moraga, A. (2001). Consumo de alcohol entre los médicos de Atención Primaria. Atención Primaria, 28, 259-262.

Rodríguez Ibáñez, M., Pérez Trullén, A., Clemente Jiménez, M., Herrero Labarga, I., Rubio Arribas, V. y Sampedro Martínez, E. (2001). Prescripción facultativa o consejo médico antitabaco en atención primaria: opiniones desde el otro lado de la mesa. Archivos de Bronconeumología, 37, 241-246.

Rosta, J. (2008). Hazardous alcohol use among hospital doctors in Germany. Alcohol and alcoholism (Oxford, Oxfordshire), 43, 198203. doi:10.1093/alcalc/agm 180

Rosta, J. y Aasland, O. G. (2005). Female surgeons' alcohol use: a study of a national sample of norwegian doctors. Alcohol and alcoholism (Oxford, Oxfordshire), 40, 436-440. doi:10.1093/alcalc/agh186

Rosta, J. y Aasland, O. G. (2010). Age differences in alcohol drinking patterns among Norwegian and German hospital doctors--a study based on national samples. German medical science: GMS e-journal, 8, Doc05. doi:10.3205/000094

Scal, P., Hennrikus, D., Ehrlich, L., Ireland, M. y Borowsky, I. (2004). Preparing residents to counsel about smoking. Clinical pediatrics, 43, 703-708. doi:10.1177/000992280404300803

Smith, D. R. y Leggat, P. A. (2007). An international review of tobacco smoking in the medical profession: 1974-2004. BMC Public Health, 7, 115. doi:10.1186/1471-2458-7-115 
Sreeramareddy, C. T., Suri, S., Menezes, R. G., Kumar, H. N. H., Rahman, M., Islam, M. R.,...Vaswani, V.R. (2010). Self-reported tobacco smoking practices among medical students and their perceptions towards training about tobacco smoking in medical curricula: A cross-sectional, questionnaire survey in Malaysia, India, Pakistan, Nepal, and Bangladesh. Substance abuse treatment, prevention, and policy, 5, 29. doi:10.1186/1747-597X-5-29

Sullivan, L. E., Tetrault, J. M., Braithwaite, R. S., Turner, B. J. y Fiellin, D. A. (2011). A meta-analysis of the efficacy of nonphysician brief interventions for unhealthy alcohol use: implications for the patient-centered medical home. The American journal on addictions / American Academy of Psychiatrists in Alcoholism and Addictions, 20,343-356. doi:10.1111/j.1521-0391.2011.00143.x

Talley, B., Mary Gee, R., Allen, D., Marshall, E. S., Encinas, K. y Lim, S. (2011). Assessment of smokeless tobacco use in the history and physical examination by primary healthcare providers. Journal of the American Academy of Nurse Practitioners, 23, 443-447. doi:10.1111/j.1745-7599.2011.00631.x

Tremblay, M., Gervais, A., Lacroix, C., O'Loughlin, J., Makni, H. y Paradis, G. (2001). Physicians Taking Action Against Smoking: an intervention program to optimize smoking cessation counselling by Montreal general practitioners. CMAJ: Canadian Medical Association journal, 165, 601-607.

Ulbricht, S., Klein, G., Haug, S., Gross, B., Rumpf, H.-J., John, U. y Meyer, C. (2011). Smokers' expectations toward the engagement of their general practitioner in discussing lifestyle behaviors. Journal of health communication, 16, 135-147. doi:10.1080/10810730.2010.5 32295

Voigt, K., Twork, S., Mittag, D., Göbel, A., Voigt, R., Klewer, J.,...Bergmann, A. (2009). Consumption of alcohol, cigarettes and illegal substances among physicians and medical students in Brandenburg and Saxony (Germany). BMC Health Services Research, 9, 219. doi:10.1186/14726963-9-219

Wallace, A. E., Sairafi, N. A. y Weeks, W. B. (2006). Tobacco cessation counseling across the ages. Journal of the American Geriatrics Society, 54, 1425-1428. doi:10.1111/j.1532-5415.2006.00843.x 